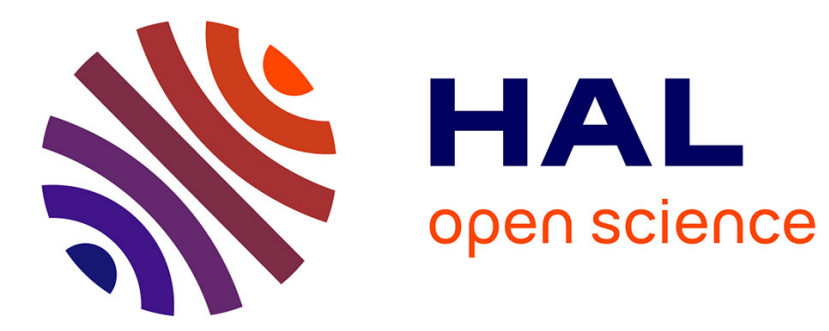

\title{
Surface oscillations and slow crack growth controlled by creep dynamics of necking instability in a glassy film
}

Pierre-Philippe Cortet, Loïc Vanel, Sergio Ciliberto

\section{To cite this version:}

Pierre-Philippe Cortet, Loïc Vanel, Sergio Ciliberto. Surface oscillations and slow crack growth controlled by creep dynamics of necking instability in a glassy film. European Physical Journal E: Soft matter and biological physics, 2008, 27 (2), pp.185. 10.1140/epje/i2008-10370-y . ensl-00295166v2

\section{HAL Id: ensl-00295166}

https://hal-ens-lyon.archives-ouvertes.fr/ensl-00295166v2

Submitted on 12 Mar 2018

HAL is a multi-disciplinary open access archive for the deposit and dissemination of scientific research documents, whether they are published or not. The documents may come from teaching and research institutions in France or abroad, or from public or private research centers.
L'archive ouverte pluridisciplinaire $\mathbf{H A L}$, est destinée au dépôt et à la diffusion de documents scientifiques de niveau recherche, publiés ou non, émanant des établissements d'enseignement et de recherche français ou étrangers, des laboratoires publics ou privés. 


\title{
Surface oscillations and slow crack growth controlled by creep dynamics of necking instability in a glassy film
}

\author{
Pierre-Philippe Cortet, Loïc Vanel, and Sergio Ciliberto \\ Université de Lyon, Laboratoire de physique, Ecole Normale Supérieure de Lyon, CNRS, France \\ Received : date / Revised version : date
}

\begin{abstract}
Résumé We study experimentally the slow growth of a single crack in a glassy film of polycarbonate submitted to uniaxial and constant imposed load. Flame-shaped macroscopic zones of plastic deformation appear at the tips of the crack and the formation of these plastic zones involves a necking instability. In order to understand the crack growth dynamics, we study first the growth dynamics of the plastic zones alone, i.e. without crack, at constant imposed load. We find that the growth velocity of the neck can be very well described by the same Eyring's factor than the one describing the creep flow of polycarbonate. In addition, we discover that a surface oscillation with a very large wavelength to amplitude ratio occurs during the neck propagation, and that both wavelength and amplitude are proportional to the film thickness. Finally, we succeed to model analytically the dependance of the instantaneous crack velocity with experimental variables using Dugdale-Barenblatt static description of crack tip plastic zones associated to an Eyring's law and an empirical dependence with the crack length that may come from a residual elastic field.
\end{abstract}

PACS. 46.50.+a Fracture mechanics, fatigue and cracks - 61.41.Be Polymers, elastomers, and plastics 81.40.Lm Deformation, plasticity, and creep - 68.35.Ct Interface structure and roughness

\section{Introduction}

Strength of solids is still characterized in handbooks by specifying a certain critical stress (for instance, a tensile stress) above which the solid is expected to break apart. However, it has been known since at least the 1940s 112 that a solid submitted to a subcritical stress, i.e. a stress lower than the critical one, will break anyway after a certain amount of time. This phenomenon called delayed, time-dependent or subcritical failure may have catastrophic consequences. Therefore, understanding the mechanisms of subcritical rupture of solids has been mainly a concern for engineers but has also become an important goal of fracture physics in order to improve the resistance of structures. According to reported experimental works 1234567 , the dependence of the rupture time with applied stress $\sigma$ can be described in many kinds of materials (glasses, polymers, metal alloys, semi-conductors, rocks...) by an Arrhenius law with an energy barrier decreasing with $\sigma$. This proposed universality is surprising since these materials have micro-structures and rheological properties very different from one another, and the rupture dynamics is certainly expected to be dependent on those properties. To lift this paradox, one must go beyond usual characterization based on global properties such as rupture time or essential work of fracture. Instead, it is worth studying experimentally the full time-resolved rupture dynamics, from the stress application to the final breakdown of the sample. A convenient system to start with is a two-dimensional solid with a single macroscopic initial crack submitted to a uniaxial constant load.

In this context, recent experimental studies [7] have shown that subcritical crack growth in paper sheets can be successfully described by a thermally activated mechanism inspired from previous theoretical works concerning elastic brittle media 8910. Experimental study of slow crack growth in a visco-plastic material under stress has been also a very active topic 111213. General theoretical frameworks 141516] have been proposed to predict the dependence of the crack growth velocity with experimental parameters using characteristic material time-response functions such as its compliance. However, these models involve complex integro-differential equations which are hardly tractable in practical situations where visco-plastic effects are strong. Consequently, the experimental time evolution of the instantaneous crack growth dynamics can not be captured easily by current models.

In order to provide more experimental insight in our understanding of visco-plastic effects during slow crack growth, we have performed an experimental study of the slow growth of a single crack in amorphous polymer films submitted to uniaxial and constant imposed load. The films are made of polycarbonate which is a highly nonbrittle glassy material. We observe that a large flameshaped area where the material is plastically deformed, so called the plastic zone, forms ahead of each crack tip. The formation of the plastic zone involves a necking instability that we study separately on rectangular strips without any 
cracks. We observe the growth of the necking instability at constant imposed load and show that it is determined by the same Eyring's factor than the one describing creep flow of polycarbonate. In addition, we discover that the material drawn during the plastic flow at the neck undergoes a surface instability with a wavelength and amplitude that depend on the thickness of the sample. Then, we study in detail the dynamics of crack propagation. We observe that there is some scatter in the crack dynamics due to uncontrollable effective initial experimental conditions. Nevertheless, we are able to propose an analytical expression for the instantaneous crack growth velocity as a function of experimental parameters [17. The law involves a Dugdale-Barenblatt static description of crack tip plastic zones associated to an Eyring's law and an empirical dependence with the crack length that may come from a residual elastic field.

\section{The experimental set-up and the fracture experiments}

The experiments consist in loading $125 \mu \mathrm{m}$ thick isotropic polycarbonate films with uniaxial and constant imposed stress $\sigma$. The polycarbonate films used are Bayer Makrofol ${ }^{\circledR}$ DE and have the properties of bulk material. The experimental set-up is made of a tensile machine driven by a motor (Micro Controle UE42) controlled electronically to move step by step (Micro Controle ITL09). The samples are mounted on the tensile machine with both ends attached with adhesive tape and rolled twice over rigid bars clamped on jaws. The motor controls the displacement of one jaw (400 steps per micrometer) while the other jaw is rigidly fixed to a gage (Hydrotonics-TC) which measures the force applied to the sample. The samples are loaded by increasing the distance between the jaws. A feedback loop allows to adjust the displacement in order to keep the applied force $F$ constant with a precision better than $0.5 \mathrm{~N}$ and a response time less than $10 \mathrm{~ms}$. This setup is mainly used to study the growth of cracks in films during creep tests but it allows to perform various tensile experiments.

During the experiments, we light the samples from the back and the transmitted light is collected by a high speed digital camera (Photron Ultima 1024) at a resolution of $512^{*} 1024$ pixels $^{2}$. This allows to follow the dynamical phenomenon occurring in the samples, either the growth of a crack or the growth of a plastic zone. We observe that the global deformation of the polycarbonate film during a creep experiment is correlated, at least in the accelerating phase, in a reproducible way to the growth dynamics of the object of interest (crack or plastic zone). We take advantage of this property by triggering the camera at fixed increment of sample deformation (about one micron) rather than at fixed increment in time. This avoids saturation of the onboard memory card when the phenomenon dynamics is slow and makes the acquisition rate faster when dynamics becomes faster and starts to have an effect on global deformation. We acquire around one thousand images per experiment.
The main experiments presented in this paper consist in the growth of a single linear crack in a polycarbonate film submitted to uniaxial and constant imposed force. Before each experiment, a crack of length $\ell_{i}$ is initiated at the center of the polycarbonate sample (height $H=21 \mathrm{~cm}$ (same direction as the crack), length $L=24 \mathrm{~cm}$, thickness $e=125 \mu \mathrm{m})$ using calibrated blades of different lengths (from 0.5 to $3 \mathrm{~cm}$ ). Then, a constant force $F$ (from 750 to $1000 \mathrm{~N}$ ) is applied to the film perpendicularly to the crack direction, so that we get a mode 1 crack opening configuration. Using the camera, we follow the growth of the fracture and its process zones, under constant applied stress $\sigma=F / e H$ until the total rupture of the sample. The applied stress $\sigma$ is chosen such that crack growth is slow, i.e. smaller than a critical one $\sigma_{c}$, above which crack propagation occurs in a few seconds.

\section{Basic mechanical properties of polycarbonate films}

In order to characterize the material in which the crack will grow, we perform some preliminary experiments on polycarbonate films (height $21 \mathrm{~cm}$, length $24 \mathrm{~cm}$ ), without crack, submitted to uniaxial deformation at a constant deformation rate. A typical experimental stress-strain curve is presented in Fig. [1 The polymer films show the classical behavior of an elasto-plastic material with a quasielastic behavior for small strains followed by a bell profile and a plateau. It is well-known that the plateau is followed for larger strains (not shown in Fig. 团) by a strainhardening curve up to rupture. The different characteristic values observed on this graph are in good agreement with the ones measured by Lu and Ravi-Chandar in bulk polycarbonate [18. For a strain rate of $1.9310^{-4} \mathrm{~s}^{-1}$, we measure the experimental values of the maximum reachable stress $\sigma_{p}=5.210^{7} \mathrm{~N} . \mathrm{m}^{-2}$, the plastic plateau stress $\sigma_{\text {plat }}=4.4510^{7} \mathrm{~N} \cdot \mathrm{m}^{-2}$, and the Young modulus for small strains $Y=1.9410^{9} \mathrm{~N} . \mathrm{m}^{-2}$.

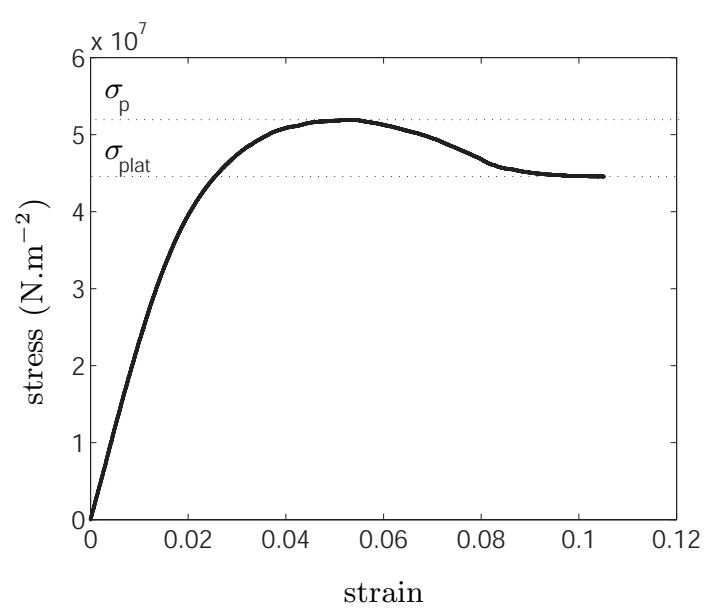

Fig. 1. Stress as a function of strain for a $125 \mu \mathrm{m}$ thick polycarbonate film loaded with a $1.9310^{-4} \mathrm{~s}^{-1}$ strain rate. 


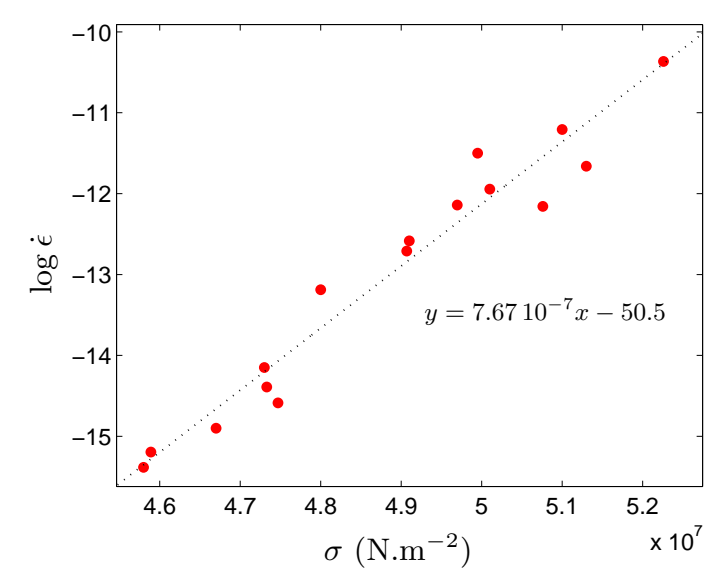

Fig. 2. Natural logarithm of the strain rate of polycarbonate strips as a function of the applied stress during creep experiments and the corresponding linear fit of the data.

As polycarbonate is a visco-plastic material, there is a strong dependence of the previous stress-strain curve on the strain rate that corresponds to the viscous properties of the material. To quantify these properties, we performed creep experiments on polycarbonate film rectangular strips (height $2 \mathrm{~cm}$, length $24 \mathrm{~cm}$ ) and measure the strain rate of the material as a function of the applied stress during the stationary creep regime. We can see in Fig. 2 that there is an exponential dependence of the strain rate $\dot{\epsilon}$ on the applied stress $\sigma$ according to :

$$
\dot{\epsilon}=\dot{\epsilon_{1}} e^{a \sigma}
$$

with $\dot{\epsilon}_{1}=1.1710^{-22} \mathrm{~s}^{-1}$ and $a=7.6710^{-7} \mathrm{~m}^{2} . \mathrm{N}^{-1}$. Actually, polycarbonate creep is known to obey an Eyring's law [19] relating its strain rate $\dot{\epsilon}$ to the applied stress $\sigma$ and the temperature $T$ [20] :

$$
\dot{\epsilon}=\dot{\epsilon_{1}} e^{\frac{V \sigma}{k_{B} T}}
$$

Then, we are able to estimate the corresponding characteristic activation volume $V=3.1010^{-27} \mathrm{~m}^{3}=(1.46 \mathrm{~nm})^{3}$ of our polycarbonate. Strictly speaking, the Eyring's law involves a sinh function rather than an exponential one, but given the value of the activation volume and the applied stress range, the exponential function is a very good approximation.

\section{The flame-shaped plastic zone ahead of the crack tip}

In each crack growth experiment, during the loading phase of the film, a macroscopic flame-shaped plastic zone appears at each tip of the crack and grows with the applied stress (cf. Fig. 3 where are defined $\ell$, the crack length and, $\ell_{\mathrm{pz}}$ the plastic zone length from tip to tip). This zone was previously observed by Donald and Kramer [21]. In the late loading stage, the crack may also start to grow at a time that appears to be statistical. It is probably a consequence of the dispersion in the local toughness of the

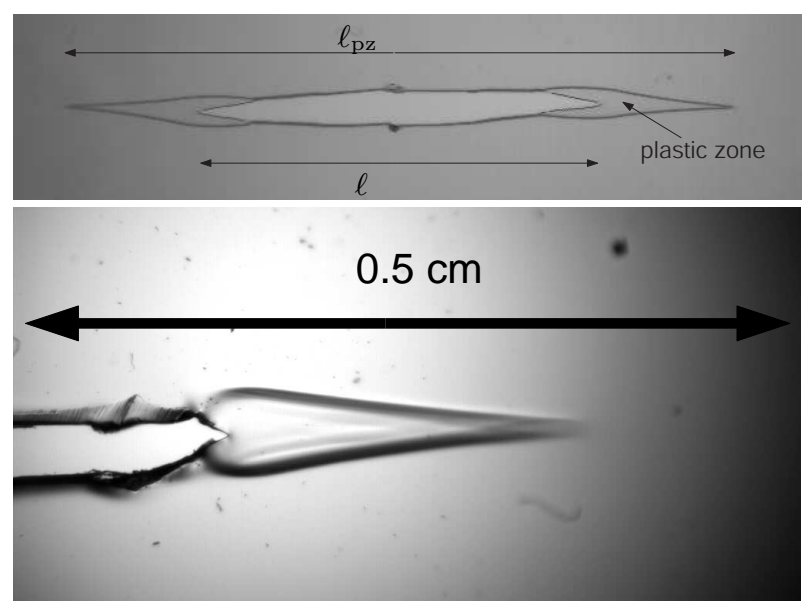

Fig. 3. On top : image of a crack in a polycarbonate film with its macroscopic plastic zone at each tip. $\ell$ is the crack length and $\ell_{\mathrm{pz}}$ is the plastic zone length from tip to tip. At the bottom : plastic zone at the tip of a growing crack in a polycarbonate film.

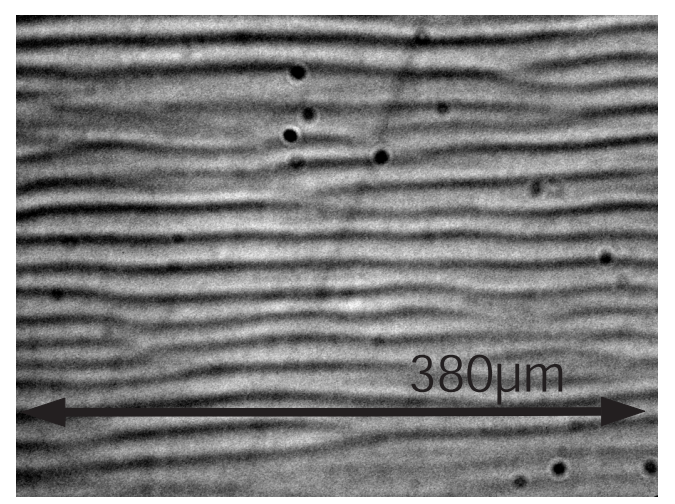

Fig. 4. Microscopic image in the plastic zone showing striations quasi-parallel to the crack direction.

material or in the initial crack tip shape. Consequently, the real experimental initial condition, obtained when the constant stress $\sigma$ is reached, is not exactly $\ell=\ell_{i}$. Depending on the moment when the crack starts to grow during the loading phase, the true initial condition of the creep experiment will be a couple of values for the crack and plastic zone length : $\left(\ell^{*}, \ell_{\mathrm{pz}}^{*}\right)$. We will see that the statistical nature of this couple is probably the explanation for all the statistics in the crack growth dynamics observed during the fracture experiments. Finally, during the imposed stress stage, the plastic zones and the crack are both growing until the final breakdown of the sample in a way that the crack never catches up the plastic zone tip.

Inside the plastic zone, the film is subjected to a thinning which brings its thickness from $125 \mu \mathrm{m}$ to about $75 \pm$ $5 \mu \mathrm{m}$ (measured on post-mortem samples). This thinning corresponds to a phenomenon called necking that usually comes with the plasticization of amorphous materials. On microscopic images (cf. Fig. 4), one can see in the plastic zone the presence of striations quasi-parallel to the fracture direction with a spatial periodicity of $26 \pm 3 \mu \mathrm{m}$. In order to understand the dynamics of crack growth in 
polycarbonate films, it is necessary to obtain more informations about the mechanical and dynamical behavior of the plastic zones that appear at the crack tips.

\section{Macroscopic plastic zones formed by necking instability}

\subsection{Plastic zone growth dynamics}

The kind of plastic zone that we observe at the tips of a crack in polycarbonate films is not specific to the fracture phenomenon. Indeed, the same kind of zone can also be observed in polycarbonate film samples without crack during creep or traction experiments. These zones actually initiate on defects of the sample. In order to better understand the dynamics of these plastic zones, we performed creep experiments on polycarbonate rectangular strips (height $1.5 \mathrm{~cm}$, length $24 \mathrm{~cm}$ ) that were previously damaged in their central part (cf. Fig. 5 (a)). Actually, a damage line is created by sliding a hard but smooth object in slight compression against the strip along its width direction. During the loading stage of the creep tests, the damage line triggers a necking instability (i.e. a plastic deformation accompanied by a thinning of the material cross-section, see Fig. [5 (c)) and extends into a well-defined band-shaped plastic zone. Once the constant stress is reached, i.e. during the creep stage, the plastic zone is growing as we can see in Fig. 馬(b).

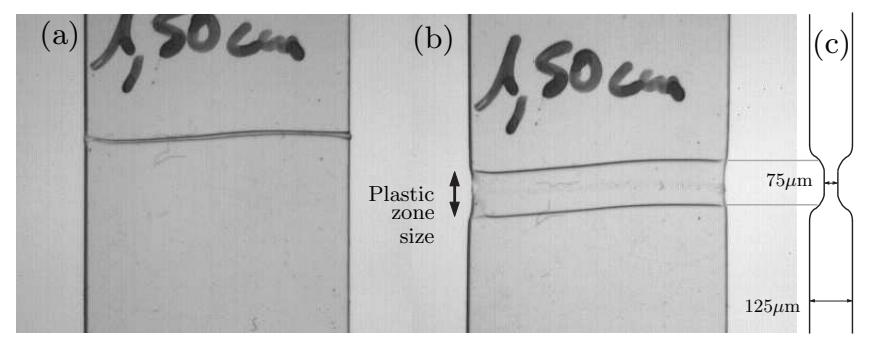

Fig. 5. Image of a plastic zone in a polycarbonate strip during a creep test, at an early stage (a) and later on when the zone has developed (b). (c) Schematic view of the strip thickness profile.

As it is easily understood, the increase in plastic zone size can be the result of two simultaneous processes : the creep of the material in the plastic zone and the transition of new material into the plastic phase through the elasto-plastic frontier i.e. the shoulder of the neck. To discriminate between these two processes, we performed Particle Imaging Velocimetry (PIV) measurements during the same creep experiments as those described in the previous paragraph. Actually, before each experiment, the samples were coated with a cloud of glass beads (diameter $=100 \mu \mathrm{m}$ ) on their surface. Then, the PIV technique allowed us to measure the field of the local velocity in the area of the plastic zone. As we can see in Fig. [6] the material in the neck between the two shoulders is submitted to a strain

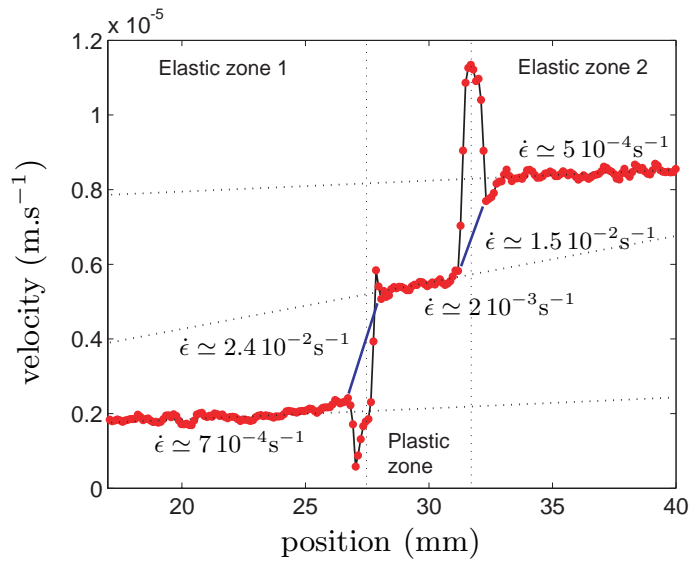

Fig. 6. Velocity of the surface of the sample as a function of the position in the area of a plastic zone in the direction normal to the elasto-plastic frontier. These PIV data correspond to the creep experiments illustrated in Fig. 5 . The two peaks observed at the frontiers of the plastic zone are actually artefacts of the PIV technique due to the misleading motion of the black lines that materialize optically the elasto-plastic frontiers, i.e. the shoulders of the neck, and do not move with the bulk material.

rate 3 times larger than the elastic material outside. Moreover, when the material crosses the elasto-plastic frontier, it is submitted to a strain rate 10 times larger than the material in the neck. Finally, when we look at the cumulative effects of these different strain rates, we conclude that the material undergoes a large strain of about $50 \%$ when it is drawn into the neck, which is much larger than the strain cumulated during the whole experiment, between $1 \%$ and $5 \%$, away from the shoulders of the neck. So, the displacement of the elasto-plastic frontier, and therefore the growth of the plastic zone, is mainly due to the migration of material from the elastic to the plastic zone and not to the simple creep of the material in the neck.

The time-evolution of the necking instability for a constant applied stress can be characterized by measuring the plastic zone size $L(\sigma, t)$ between the two shoulders (see Fig. 5). As one can see in Fig. 7(a), the plastic zone growth accelerates with time. It has proved difficult to describe analytically the time dependence of the plastic zone size $L(\sigma, t)$. However, we succeeded to rescale the growth curves for different applied stresses using the following law (cf. Fig. [7(b)) :

$$
L(\sigma, t)=f(\eta(\sigma) t)
$$

where $L$ is the plastic zone size, $t$ the current time and $\eta$ a constant defined with an arbitrary prefactor in order to make the growth curves collapse. It means that the band growth velocity can be written as :

$$
v(\sigma, t)=\eta(\sigma) f^{\prime}(\eta(\sigma) t) .
$$

Hence, the initial growth rate $v_{i}=\eta(\sigma) f^{\prime}(0)$ of the plastic zone characterizes fully the influence of the applied stress $\sigma$ on the growth dynamics.

In Fig. 8 is plotted the natural logarithm of $v_{i}$ as a function of $\sigma$. We can see that the initial growth rate $v_{i}$ 

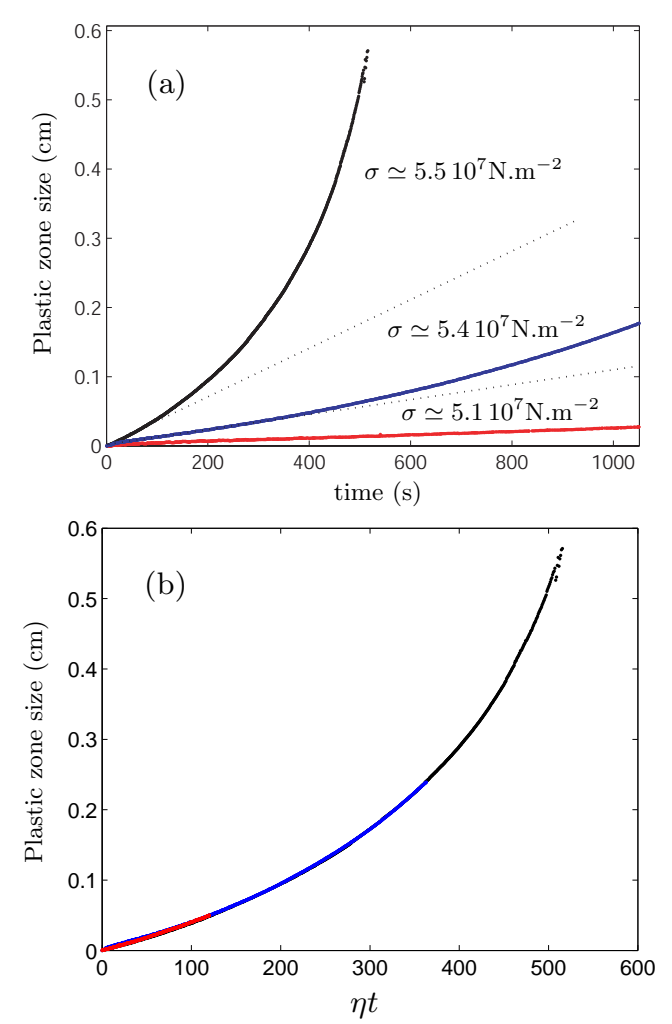

Fig. 7. Plastic zone size as a function of (a) time and (b) rescaled time for three experiments performed with different applied stresses. Constant $\eta$ is defined with an arbitrary prefactor in order to make the growth curves collapse.

of the plastic zone diverges dramatically with the applied stress $\sigma$. These data appear to fit quite well with a linear law. It means that a good description of the plastic zone growth rate dependence with $\sigma$ is the exponential law :

$$
v_{i}=v_{0} e^{a_{\text {plast }} \sigma}\left(=v_{0} e^{\frac{V_{\text {plast } \sigma}}{k_{B} T}}\right)
$$

with $a_{\text {plast }}=7.4110^{-7} \mathrm{~m}^{2} . \mathrm{N}^{-1}$ (i.e. $V_{\text {plast }}=3.0010^{-27} \mathrm{~m}^{3}=$ $\left.(1.44 \mathrm{~nm})^{3}\right)$ and $v_{0}=4.810^{-24} \mathrm{~m} \cdot \mathrm{s}^{-1}$. Such kind of law corresponds well to the Eyring's law that describes the creep rate dependence with stress and temperature for polymers. It is important to highlight that the prefactor $a_{\text {plast }}$ of the stress in this law is very close to the one in Eq. (II). It suggests that these two prefactors can actually constitute the same material constant of polycarbonate $V / k_{B} T$ and that the dynamics of the necking instability is controlled by the intrinsic creep properties of polycarbonate away from the neck region.

\subsection{Fine structural properties of the plastic zones}

The plastic deformation zones that are observed in polycarbonate films correspond to a necking phenomenon with a thinning of the material by a factor of $0.60 \pm 0.05$. When one observes these zones with a microscope, one can also see striations (very similar to the ones in Fig.

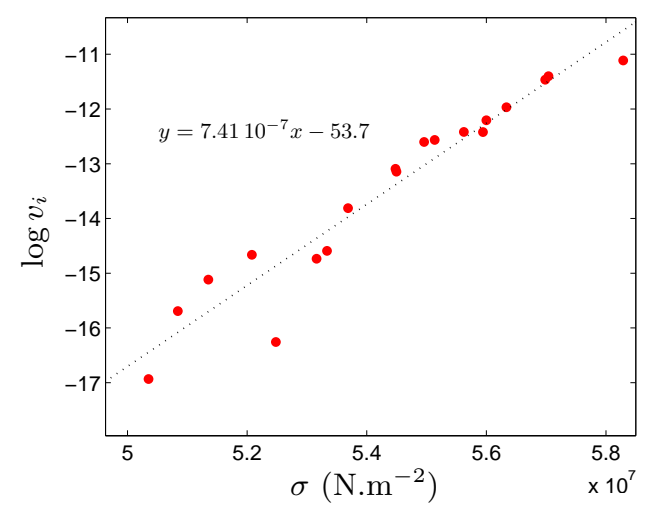

Fig. 8. Natural logarithm of the plastic zone initial velocity as a function of the applied stress. The dotted line is a linear fit.

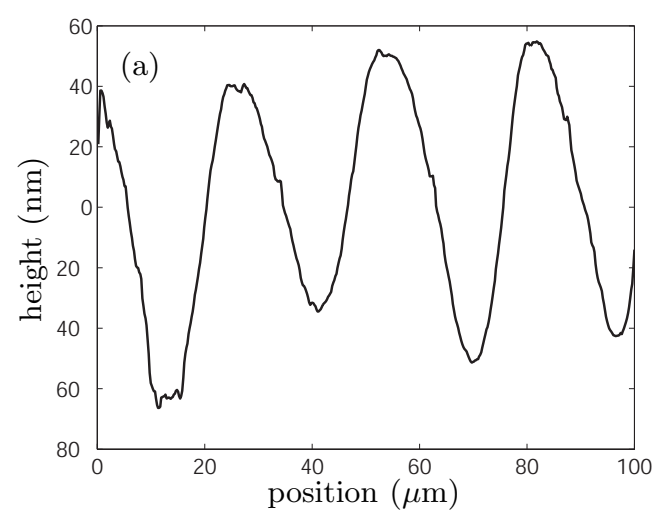

(b)

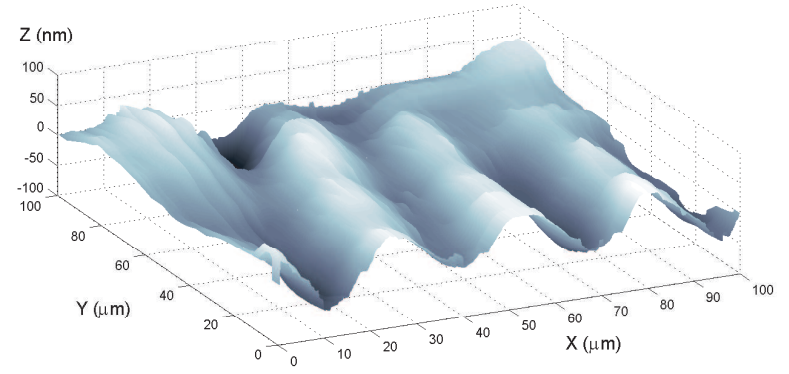

Fig. 9. (a) AFM profile of the plastic zone surface perpendicularly to the striations, (b) AFM three dimensional plot of a plastic zone surface, for a crack tip plastic zone in a $125 \mu \mathrm{m}$ thick film.

4) that are perpendicular to the elastic-plastic frontier growth direction at the time they are created. Using atomic force microscopy, we prove these striations to be the result of surface oscillations of the film of about $100 \mathrm{~nm}$ (for $125 \mu \mathrm{m}$ thick films) peak to peak amplitude as we can see in Fig. 9 Periodic patterns formed during the necking instability of polymers have already been reported in the literature. For instance, stress-oscillations attributed to thermo-mechanical effects can leave periodic traces in drawn polymers 2223] but the observed wavelengths are a priori too large to explain the surface oscillations in our experiments. 


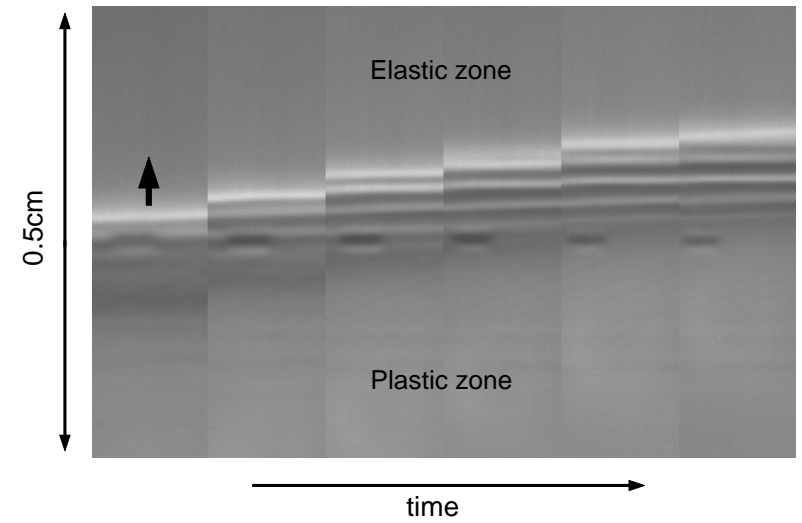

Fig. 10. Spatiotemporal microscopic image of the elasticplastic frontier area during a creep experiment in a $125 \mu \mathrm{m}$ thick polycarbonate strip. This figure shows the in situ formation of the surface oscillations inside the frontier, i.e. the shoulder of the necking instability, as it moves.

The surface oscillations of the film are created inside the frontier between the elastic and plastic zone when this one moves (cf. Fig. 10). It is important to notice that their amplitude of about $100 \mathrm{~nm}$ is very small. It is actually thousand times smaller than the initial thickness of the films that is $125 \mu \mathrm{m}$. In contrast, the wavelength of these oscillations, $\lambda=26 \pm 3 \mu \mathrm{m}$, is of the same order of magnitude as the thickness. The wavelength and the amplitude of the oscillations are probably slightly dependent on the local stress or strain rate when they are created but this dependence is very fine and we did not study it. However, the dependence with the initial thickness of the film was studied. We made additional experiments with films that have a thickness of $250 \mu \mathrm{m}$ or $375 \mu \mathrm{m}$. The wavelength and the amplitude of the oscillations appear to be proportional to the initial film thickness $e$ with : $\lambda=(0.20 \pm 0.02) e$ and amplitude $=(8 \pm 1) 10^{-4} e$.

It would be interesting to understand the physical mechanisms responsible for the formation of these oscillatory patterns. However, the consequences on the growth dynamics of the plastic zone are probably minor and we will not go in such a detailed description of the visco-plastic dynamics in the rest of the paper.

\section{Crack growth curves}

Typical growth curves of the fracture and plastic zone are shown in Fig. 111(a). Both curves show a quite similar smooth shape 24]. We observe, once the loading phase is finished, at the beginning of the constant stress phase, large velocities of the fracture and the plastic zone tips which decrease till reaching quasi-constant values before increasing back dramatically until the final rupture at time $T_{r}$.

The regular shape of the growth curves let us think that the crack growth in polycarbonate films is a deterministic phenomenon. However, as one can see in Fig. 111(b), for identical experimental conditions i.e. same initial crack
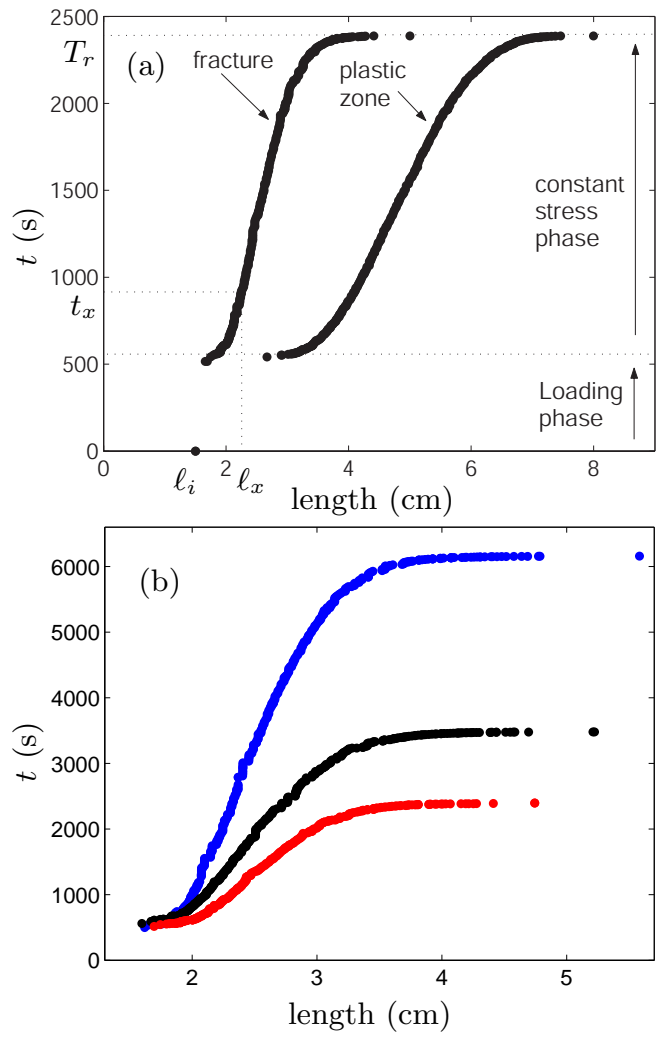

Fig. 11. (a) Time as a function of both the crack and plastic zone lengths for an imposed stress experiment $\left(\ell_{i}=1.5 \mathrm{~cm}\right.$, $F=900 \mathrm{~N})$. We indicate the position of the inflexion point $t_{x}, \ell_{x}$ of the crack growth curve. (b) time as a function of $\ell$ for three experiments performed with the same experimental conditions $\left(\ell_{i}=1.5 \mathrm{~cm}, F=900 \mathrm{~N}\right)$.

length and same applied stress, we notice a large dispersion of the rupture times and more generally of the crack growth dynamics. There is actually up to a factor five between the rupture time of the fastest and slowest experiments.

We suggest that the explanation for this statistics in the crack growth dynamics does not come from the growth mechanism itself, but is a consequence of the dispersion in the effective initial conditions at the beginning of the constant stress phase of the experiment $\left(\ell^{*}, \ell_{\mathrm{pz}}^{*}\right)$. These initial conditions are clearly statistical and hardly controllable in our experiment. They are dependent on the moment when the crack starts growing during the loading stage of the sample and they determine all the rest of the experiment. The initial growth velocity of the crack is probably correlated with the stress at the border of the sample at the time it starts growing.

A detailed study of the probability density function of the rupture time and the effective initial conditions has not been performed since it would need a huge amount of experiments for each experimental conditions. Then, in the next sections, we will first look at the average behavior (temporal average over an experiment and statistical average over series of experiments) of the dynamics as a function of the applied stress. We will try to understand 
better the mechanisms in action in the phenomenon of crack growth in polycarbonate films and finally infer a meaningful instantaneous crack growth law.

\section{Rupture time}

In this section, we study the dependence of the rupture time $T_{r}$ of the polycarbonate samples as a function of the applied stress $\sigma$ for a given initial crack length $\ell_{i}$. In Fig. [12(a), we show the evolution of the average rupture time as a function of the applied stress for a series of experiments performed for $\ell_{i}=1.5 \mathrm{~cm}$. More precisely, for each stress value, we show the rupture time $\left\langle T_{r}\right\rangle$ averaged over ten experiments at least.
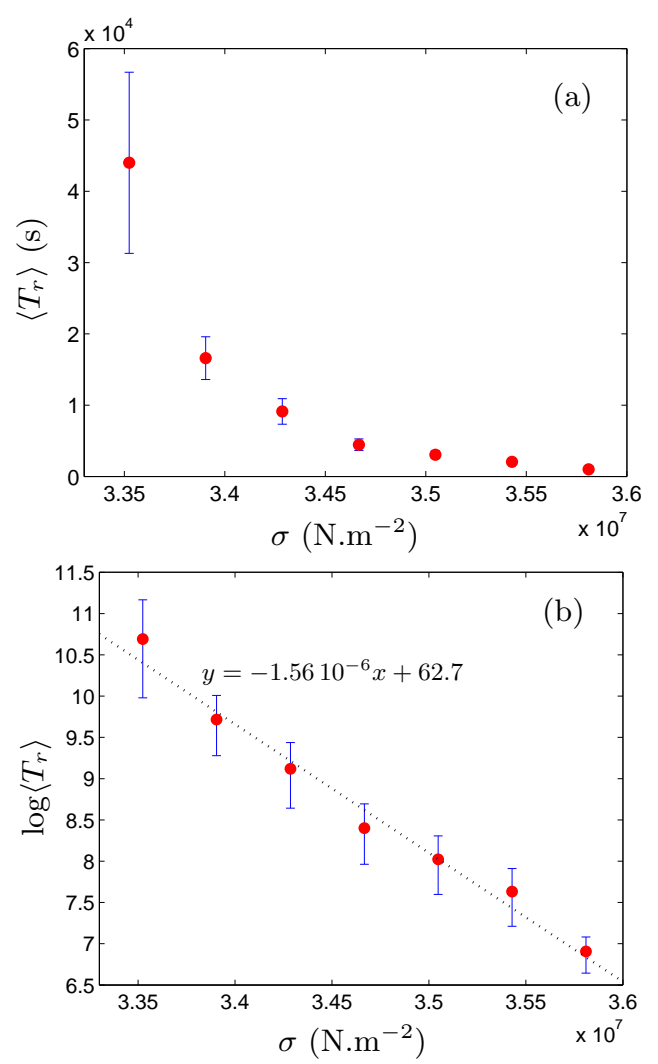

Fig. 12. Average rupture time (a) and its natural logarithm (b) as a function of the applied stress for a series of experiments performed for $\ell_{i}=1.5 \mathrm{~cm}$.

We see a dramatic dependence of $\left\langle T_{r}\right\rangle$ with the applied stress. Indeed, the rupture time ranges from a large value of $410^{4}$ seconds for a $3.3510^{7} \mathrm{~N} . \mathrm{m}^{-2}$ stress to a small value of $10^{3}$ seconds for a $3.5810^{7} \mathrm{~N} . \mathrm{m}^{-2}$ stress. It corresponds to a $97.5 \%$ variation of the rupture time for a $7 \%$ variation of the stress. This dependence suggests an exponential description of the rupture time as proposed by Zhurkov [5]. To test this description, we show on Fig. 12 (b) the natural logarithm of the rupture time as a function of the applied stress. The linear fit of the data is of quite good quality and suggests that :

$$
\left\langle T_{r}\right\rangle=T_{0} e^{-\gamma \sigma}
$$

In Zhurkov's approach, the stress dependence of rupture time is interpreted as an Eyring's law [19] with $\gamma=V_{\gamma} / k_{B} T$ where $V_{\gamma}$ is assumed to be a characteristic volume of the material. However, in our experiments, the parameter $V_{\gamma}$ can not be a constant since a different initial crack length $\ell_{i}$ gives a completely different rupture time for the same applied stress. Thus, the external applied stress $\sigma$ can not be the single control parameter of the rupture dynamics. Then, it is clear that Zhurkov's description needs to be improved to take into account the specific geometry of the problem. In particular, the stress level in the plastic zone close to the crack tips most probably participates in the dynamical processes leading to the crack growth.

\section{Dynamical stress in the plastic zone}

We will show in this section that the stress level in the plastic zone is dependent on the growth dynamics and must be understood as a dynamical stress. In order to estimate the stress level in the plastic zone, we need to characterize more precisely its dimensions.

The experimental ratio between the plastic zone length (defined in Fig. 13) $\ell_{\mathrm{pz}}$, and the crack length $\ell$, during the crack growth process, is plotted as a function of $\ell$ in Fig. [13(a) for three experiments performed with the same experimental conditions $\left(\ell_{i}=1.5 \mathrm{~cm}\right.$ and $\left.F=900 \mathrm{~N}\right)$ and in Fig. 13(b) for four experiments with different experimental conditions. It seems that this relation is not unique because curves for identical experimental conditions do not overlap each other. Through all the experiments that have been performed (between 10 and 20 for each experimental conditions), there is a dispersion of about $10 \%$ for the ratio $\ell_{\mathrm{pz}} / \ell$ for identical experimental conditions. Having a look at the corresponding rupture time for each experiment, a qualitative correlation between rupture time and the ratio $\ell_{\mathrm{pz}} / \ell$ appears clearly. The larger the rupture time $T_{r}$, the larger the $\ell_{\mathrm{pz}} / \ell$ ratio. This correlation is very understandable. Indeed, a larger rupture time corresponds to a slower crack dynamics that lets slightly more time for the plastic zone to grow at each position of the crack tip.

The relation between $\ell_{\mathrm{pz}}$ and $\ell$ at equilibrium has first been theoretically predicted by the Dugdale-Barenblatt cohesive zone model [25 26]. Dugdale considers the cohesive zone as an isotropic plastic material in which the stress is uniformly equal to the plastic yield stress $\sigma_{y}$ of the material. Assuming the non-divergence of the stress at the tip of the cohesive zone, he concludes to a zero stress intensity factor ${ }^{1}$ (SIF) at the tip of the plastic zone :

$$
K_{\mathrm{tot}}=K_{\mathrm{el}}\left(\sigma, \ell_{\mathrm{pz}}\right)+\tilde{K}\left(\sigma_{y}, \ell, \ell_{\mathrm{pz}}\right)=0
$$

1 At a close distance $r$ from a crack tip in an elastic material, the local stress behaves as $K / \sqrt{r}$, where $K$ is the stress intensity factor. 

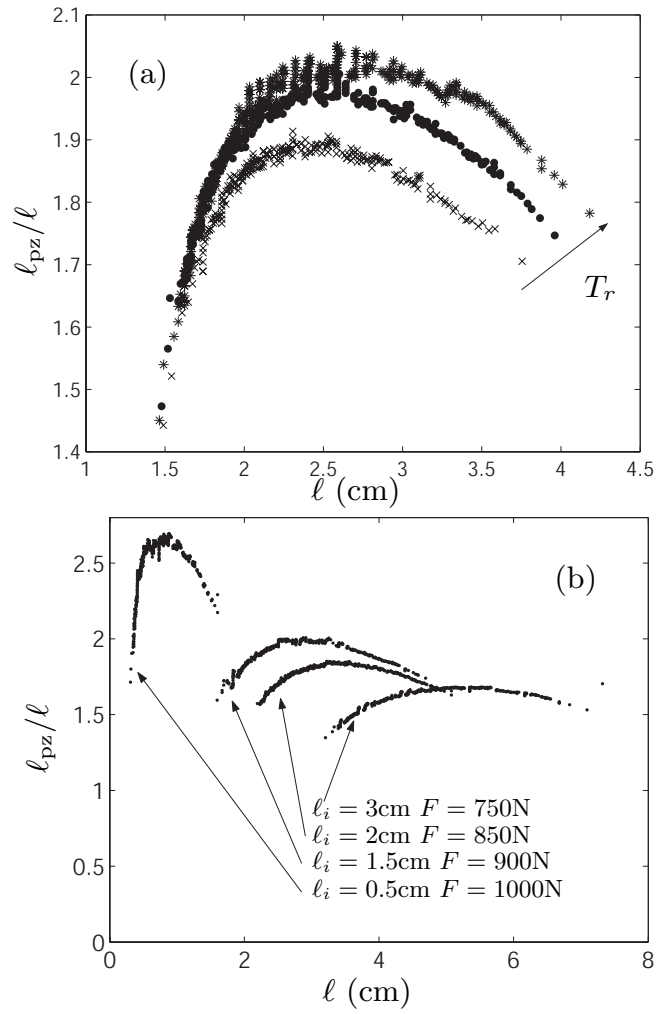

Fig. 13. $\ell_{\mathrm{pz}} / \ell$ ratio as a function of the crack length (a) for three experiments performed with the same experimental conditions $\left(\ell_{i}=1.5 \mathrm{~cm}\right.$ and $\left.F=900 \mathrm{~N}\right)$, (b) for four experiments performed with different experimental conditions.

where $K_{\mathrm{el}}$ is the traditional SIF at the tip of a crack of length $\ell_{\mathrm{pz}}$ in an elastic film submitted to $\sigma$ at its border and $\tilde{K}$ the SIF for a film fractured on a length $\ell_{\mathrm{pz}}$ and submitted only to $\sigma_{y}$ on the fracture lips between $\ell$ and $\ell_{\mathrm{pz}}$. Using analytical expressions for these SIFs in the case of an infinite elastic sample, Dugdale finds a proportionality dependence of $\ell_{\mathrm{pz}}$ on $\ell$ :

$$
\frac{\ell_{\mathrm{pz}}}{\ell}=\frac{1}{\cos \left(\frac{\pi}{2} \frac{\sigma}{\sigma_{y}}\right)} .
$$

This model was successfully faced with experimental data in metals [25].

In Fig. 13] one can see that the $\ell_{\mathrm{pz}} / \ell$ ratio is not constant during a constant stress experiment so that the $\ell_{\mathrm{pz}}$ against $\ell$ relation is not a proportionality relation. Regarding this non-proportionality, finite size corrections (corrections when $\ell$ is not negligible compared to the sample height) to the Dugdale-Barenblatt model lead to an opposite curvature [27] to the one observed experimentally. The most simple explanation for this non-proportionnality lies in the fact the crack growth is not stationary so that the yield stress can not be considered constant. Reversing the problem and using the experimental values of the ratio $\ell_{\mathrm{pz}} / \ell$ in the Dugdale-Barenblatt law allows to give an estimate of the Dugdale stress constant $\sigma_{y}$ that holds in the crack tip plastic zone at each position of the crack during an experiment :

$$
\sigma_{y}=\frac{\pi}{2} \frac{\sigma}{\operatorname{arcos}\left(\frac{\ell}{\ell_{\mathrm{pz}}}\right)}
$$

This stress value neglects possible spatial inhomogeneities of the real plastic zone stress. It is also an effective value since we do not account for the thinning of the film.

In our experiments, the estimated $\sigma_{y}$ fluctuates between $510^{7}$ and $5.610^{7} \mathrm{~N} \cdot \mathrm{m}^{-2}$. In order of magnitude, the values found here for $\sigma_{y}$ are in good agreement with the plastic peak stress $\sigma_{p}$ defined by the stress-strain curve of Fig. 1 that fluctuates between $5.210^{7}$ and $5.610^{7} \mathrm{~N} . \mathrm{m}^{-2}$ for the considered strain rates. It is also in good agreement with the stress range needed to grow plastic bands under creep condition in reasonable times (cf. subsection [5.1). So, the Dugdale-Barenblatt model predicts the correct order of magnitude for the $\ell_{\mathrm{pz}} / \ell$ ratio, using the maximum reachable stress $\sigma_{p}$ obtained from mechanical tests (cf. Fig. (1).

The fact the $\ell_{\mathrm{pz}} / \ell$ ratio is not constant throughout each single experiment clearly suggests that the yield stress $\sigma_{y}$ is not constant. Actually, we expect the yield stress to depend on the local strain rate of the material through an Eyring's law. Then, it is clear that a change in crack growth velocity will be reflected in a change of local strain rate, and thus in a change in the yield stress value.

\section{Average growth dynamics}

In this section, we show that the average crack growth dynamics is strongly correlated to the creep behavior of the plastic zone. To account for the global dynamics of a rupture experiment, a meaningful variable is the timeaveraged growth velocity on the whole experiment $\bar{v}$. The creep of the plastic zone can be characterized by the timeaveraged plastic stress $\overline{\sigma_{y}}$ which appears intuitively as a possible control parameter for the crack dynamics just like the stress intensity factor is for brittle materials.

The plastic stress $\sigma_{y}$ is computed at each moment using Eq. (9) with the instantaneous values of $\sigma, \ell$ and $\ell_{\mathrm{pz}}$. Then, it is averaged over time during each experiment. In Fig. 14] we plot the natural logarithm of $\bar{v}$ as a function of the time-averaged plastic stress $\overline{\sigma_{y}}$. Each point of this figure represents the mean behaviour over an experiment. We clearly see that the data are compatible with a linear law that predicts an exponential dependence of the timeaveraged growth velocity with the time-averaged stress in the plastic zone :

$$
\bar{v}=v_{0} e^{\tilde{\alpha} \overline{\sigma_{y}}}
$$

with $\tilde{\alpha}=6.310^{-7} \mathrm{~m}^{2} \cdot \mathrm{N}^{-1}$ and $v_{0}=7.810^{-21} \mathrm{~m} \cdot \mathrm{s}^{-1}$. Note that inside a series of experiments performed for the same experimental conditions (given symbol in Fig. 14), rather large variations of $\overline{\sigma_{y}}$, about $0.2510^{7} \mathrm{~m}^{2} . \mathrm{N}^{-1}$, are observed. The variations of the instantaneous value of $\sigma_{y}$ during one single experiment are even larger, up to $10^{7} \mathrm{~m}^{2} . \mathrm{N}^{-1}$ (see section 10). 


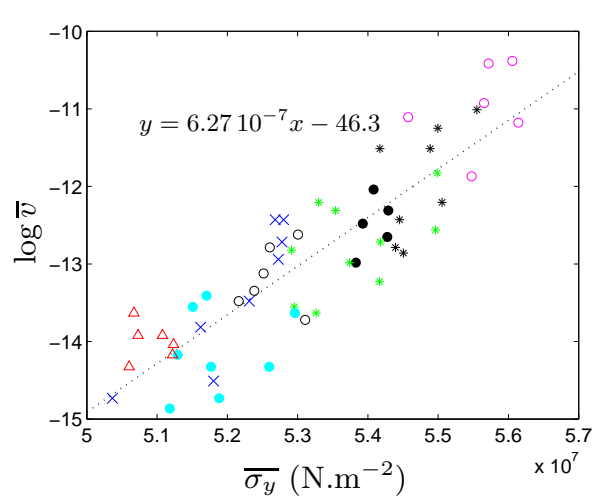

Fig. 14. Natural logarithm of the average crack growth velocity $\bar{v}$ as a function of the average plastic stress during the growth. Each point represents the average dynamical behavior during an experiment. Experimental conditions are various $\left(\ell_{i}=1.5,2,3 \mathrm{~cm}\right.$ and $\left.2.9<\sigma<3.810^{7} \mathrm{~N} \cdot \mathrm{m}^{-2}\right)$. Each experimental condition corresponds to different symbols.

It is striking that the prefactor $\tilde{\alpha}=6.310^{-7} \mathrm{~m}^{2} . \mathrm{N}^{-1}$ of the stress in the exponential law of Eq. (10) is rather close quantitatively to the one obtained for the creep growth of the plastic zone in polycarbonate bands (see section [5.1), and even to the one that describes polycarbonate creep (see Eq. (2)), particularly if we consider the dispersion of the data. It is tempting to say that both prefactors $\tilde{\alpha}$ and $a$ are coming from the same material mechanical constant of polycarbonate, $V / k_{B} T$, appearing in polycarbonate Eyring's law. Actually, we have to remind the reader that $\sigma_{y}$ is far from being constant during an experiment. It is then clear that taking the average value of $\sigma_{y}$ over an experiment constitutes a rude averaging. This could be the explanation for the $15 \%$ difference between the values of the stress prefactors in the exponential laws. With these source of imprecision in mind, we are tempted to say that the Eyring's law ruling the polymer creep might play an important role in the mechanisms of crack growth in polycarbonate films.

\section{A tentative growth law for the instantaneous dynamics}

In this section, we go beyond a simple analysis of the average growth dynamics by looking at the dependence of the crack velocity with the stress in the plastic zone at each time during the crack growth. We plot in Fig. 15(a) the instantaneous crack velocity $v=d \ell / d t$ as a function of the instantaneous value of the Dugdale-Barenblatt stress $\sigma_{y}$ during a typical experiment performed for $\ell_{i}=1.5 \mathrm{~cm}$ and $F=900 \mathrm{~N}$. We see that the description of the data by the exponential law (dotted line) from the data fit of Fig. 14 is only very approximative even if there seems to be a tendency to follow such a law, especially during the early stage of the experiment. Introducing a correction in the Dugdale-Barenblatt stress that is linear with the crack length $\ell$ allows us to collapse the experimental data on a straight line (cf. Fig. 15(b)). Actually, the correction can
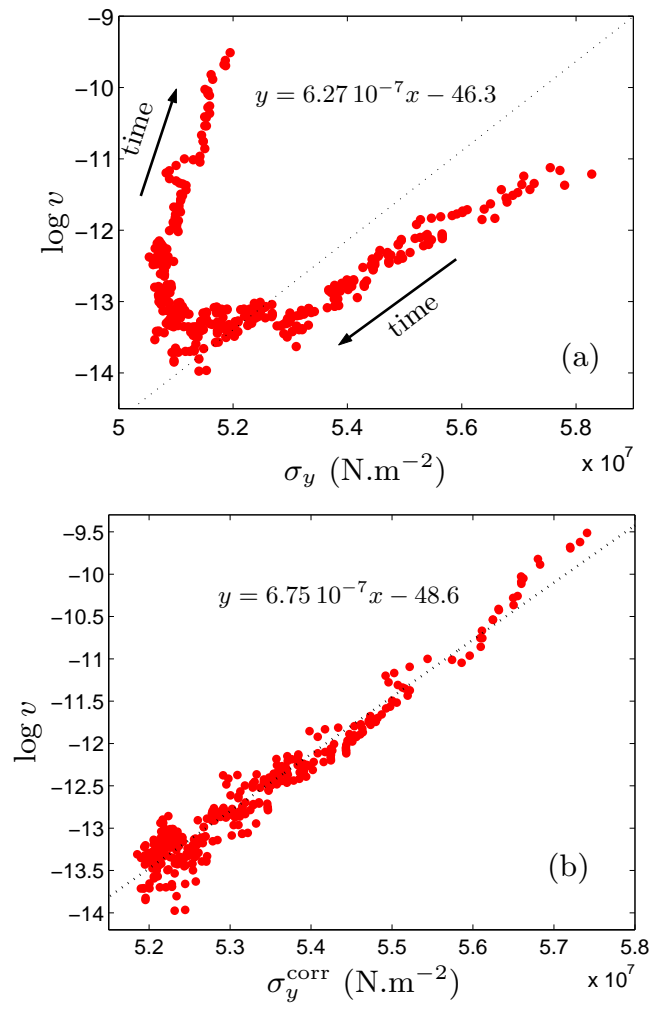

Fig. 15. Natural logarithm of the instantaneous crack growth velocity as a function of (a) the Dugdale-Barenblatt stress and (b) the corrected Dugdale-Barenblatt stress $\sigma_{y}^{\text {corr }}$ for an experiment performed with $F=900 \mathrm{~N}$ and $\ell_{i}=1.5 \mathrm{~cm}$. In Fig. (a), the dotted line is the result of the Fig. 14 data fit. In Fig. (b), the dotted line is the result of a linear data fit.

be written as :

$$
\sigma_{y}^{\text {corr }}=\frac{\pi}{2} \frac{\sigma}{\operatorname{arcos}\left(\frac{\ell}{\ell_{\mathrm{pz}}}\right)}+\kappa\left(\ell-\ell_{x}\right)
$$

with $\kappa=3.810^{8} \mathrm{~N} \cdot \mathrm{m}^{-3}$ in this case. In this equation, we introduce the crack length $\ell_{x}$ corresponding to the inflexion point in the growth curve as defined in Fig. [1] This length also corresponds to the minimum crack velocity. It is quite natural to do so since the dynamics around $\ell=\ell_{x}$ is characteristic of the time-averaged dynamics that has been revealed to approximately follow an Eyring's law in section 9

In Fig. 16](a), we plot the instantaneous crack growth velocity as a function of the stress $\sigma_{y}$ for eight experiments performed with various experimental conditions. For each experiment, we determine the value $\kappa=(3.4 \pm$ 0.6) $10^{8} \mathrm{~N} \cdot \mathrm{m}^{-3}$ that allows us to collapse each curve on a straight line. The dispersion of $\kappa$ values seems to be statistical as no systematic dependence with $\sigma$ or $\ell_{i}$ could be found. Finally, in Fig. [16 (b), we obtain a collapse of all the data when plotting as a function of $\sigma_{y}^{\text {corr }}$. This rescaling means that the crack growth velocity seems to follow :

$$
v=v_{0} e^{\alpha \sigma_{y}^{\text {corr }}}
$$

where $\alpha=6.810^{-7} \mathrm{~m}^{2} \cdot \mathrm{N}^{-1}$. Note that the collapse of the data for various experimental conditions (applied stress 

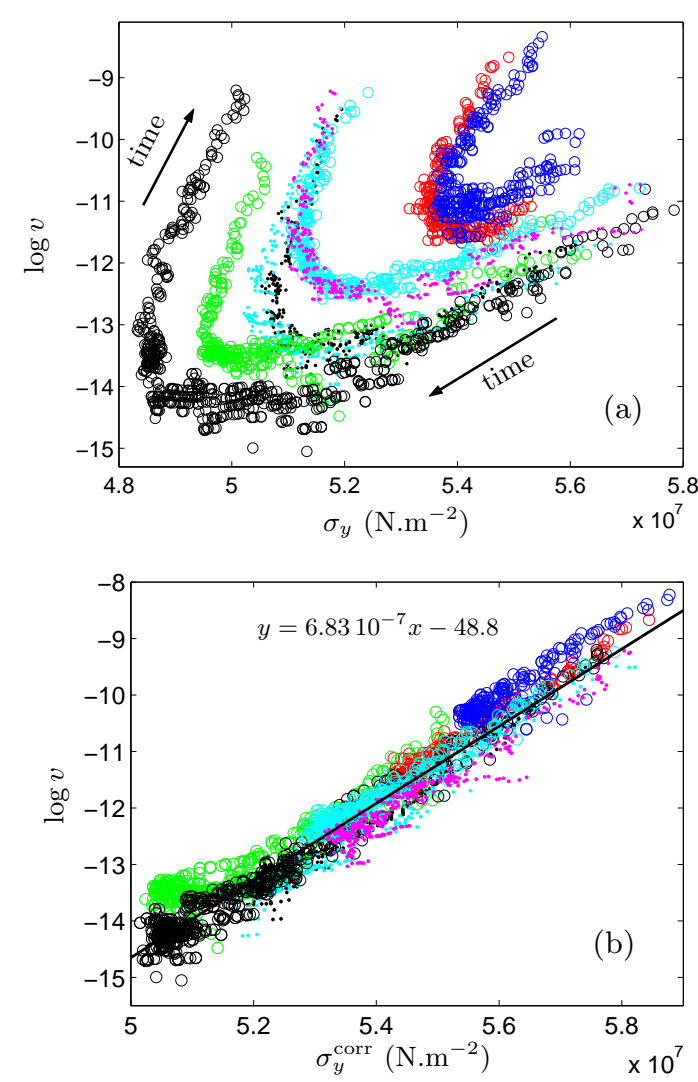

Fig. 16. Natural logarithm of the instantaneous crack growth velocity as a function of (a) the Dugdale-Barenblatt stress, (b) the corrected Dugdale-Barenblatt stress $\sigma_{y}^{\text {corr }}$ according to Eq. (11) for eight experiments performed with various experimental conditions $\left(\ell_{i}=1.5,2,3 \mathrm{~cm}\right.$ and $\left.2.9<\sigma<3.810^{7} \mathrm{~N} . \mathrm{m}^{-2}\right)$. In Fig. (b), the black line is the result of a linear data fit.

and initial crack length) means that $v_{0}$ can be considered as a constant.

In Eq. (11), the crack length at the inflexion point in the growth curve plays a particular role. It turns out that its value depends on the experimental conditions. This can be seen in Fig. 17(a) where the product $\kappa \ell_{x}$, with $\kappa=3.410^{8} \mathrm{~N} . \mathrm{m}^{-3}$, is plotted as a function of the applied stress $\sigma$. Remarkably, the dependence of $\kappa \ell_{x}$ with $\sigma$ is well approximated by a linear fit : $\kappa \ell_{x}=\sigma_{x}-\sigma$, where $\sigma_{x}=4.210^{7} \mathrm{~N} \cdot \mathrm{m}^{-2}$ is only an estimate of $\sigma$ for $\kappa l_{x}=0$.

A way to clarify the meaning of this relation is to look at the dependence of the critical stress $\sigma_{c}$ needed to break instantaneously a sample with a crack of initial length $\ell_{i}$. In brittle materials, we would expect this critical stress to decrease in $1 / \sqrt{\ell_{i}}$ since the rupture criterion is reached when the initial stress intensity factor equals the toughness of the material $K_{c}\left[\underline{7]}: \sigma_{c} \sqrt{\pi \ell_{i} / 2}=K_{c}\right.$. For an amorphous visco-plastic material such as polycarbonate, we do not get the same functional dependence. Indeed, as we can see in Fig. 17(b), the relation between $\sigma_{c}$ and $\ell_{i}$ can be approximated by a linear equation : $\beta \ell_{i}=\sigma_{s}-\sigma_{c}$, where $\sigma_{s}=4.0710^{7} \mathrm{~N} \cdot \mathrm{m}^{-2}$ and $\beta=3.5710^{8} \mathrm{~N} \cdot \mathrm{m}^{-3}$. We note that $\kappa \simeq \beta$ and $\sigma_{x} \simeq \sigma_{s}$ and will consider these quantities to be the same material constants. So, we find that the
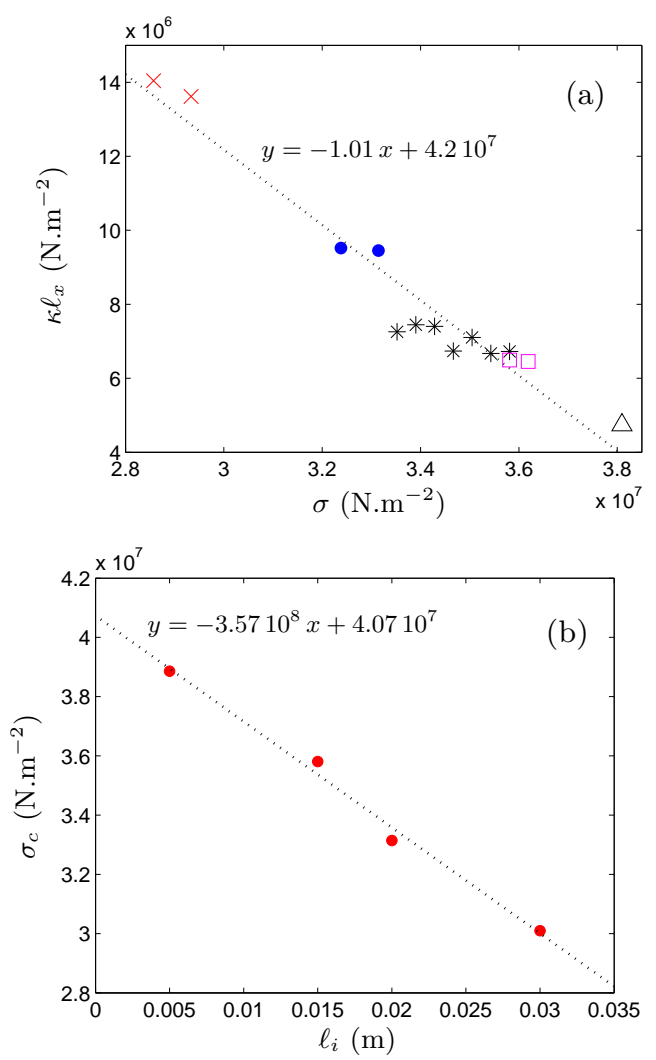

Fig. 17. (a) $\kappa \ell_{x}$ for various experimental conditions $\left(\ell_{i}=\right.$ $1.5,2,3 \mathrm{~cm}$ and $2.9<\sigma<3.810^{7} \mathrm{~N} . \mathrm{m}^{-2}$ ) as a function of the applied stress $\sigma$. (b) Critical rupture stress $\sigma_{c}$ as a function of the initial crack length $\ell_{i}$. The dotted lines are a linear fit of the data.

quantity $\Sigma\left(\sigma_{c}, \ell_{i}\right)=\sigma_{c}+\kappa \ell_{i}$ may play a role similar to the initial stress intensity factor in brittle materials. Furthermore, it allows us to interpret the value of the crack length at the inflexion point as defined by a characteristic value of the quantity $\Sigma\left(\sigma, \ell_{x}\right)=\sigma_{x} \simeq \sigma_{s}$ that corresponds to an intrinsic property of polycarbonate. Indeed, $\sigma_{s}$ is simply the value of the critical rupture stress $\sigma_{c}$ when there is no initial crack.

Finally, the effective stress $\sigma_{y}^{\text {corr }}$ may be rewritten as :

$$
\sigma_{y}^{\text {corr }}=\frac{\pi}{2} \frac{\sigma}{\operatorname{arcos}\left(\frac{\ell}{\ell_{\mathrm{pz}}}\right)}+\kappa \ell+\sigma-\sigma_{s}
$$

where we clearly see that it is composed of, the DugdaleBarenblatt estimation of the crack tip plastic zone stress $\sigma_{y}$, a linear dependence with the crack length $\kappa \ell$ and the applied stress at the borders of the sample $\sigma$. This is one of the main results of this paper. Furthermore, one can interpret the exponential dependence of the velocity as a function of $\sigma_{y}^{\text {corr }}$ (cf. Eq. 12) in a rather simple and physical way. Indeed, as we have noticed, polycarbonate creep is known to obey an Eyring's law relating its strain rate $\dot{\epsilon}$ to the applied stress $\sigma: \dot{\epsilon}=\dot{\epsilon_{1}} \exp \left(\sigma V / k_{B} T\right)$ [20]. Creep experiments performed at room temperature on our own polycarbonate samples give $V / k_{B} T=7.6710^{-7} \mathrm{~m}^{2} . \mathrm{N}^{-1}$ (see Eq. 1). The fact this prefactor $V / k_{B} T$ is of the same 
magnitude than $\alpha$ in the exponential law for the crack velocity (cf. Eq. (12)) suggests that the Eyring's law plays an important role in the mechanisms of crack growth. Additionally, it possibly means that the constant $\alpha$ of Eq. (12) identifies with the Eyring's prefactor $V / k_{B} T$. However, such a temperature dependence of $\alpha$ is still to be checked.

\section{Conclusion}

In this article, we have first presented an experimental study of the plastic deformation properties of polycarbonate films under a constant load. We have shown that the necking instability responsible for the formation of the plastic zones, such as the ones appearing at the tips of a crack, has a dynamics that follows an Eyring's like growth law. The Eyring factor appearing in the growth law for the plastic zone is almost identical to the one describing the simple creep flow of polycarbonate when there is no necking instability. It is not clear however what determines the amplitude of the velocity prefactor in the growth law. We have also discovered and characterized an a priori unknown surface instability that develops in the shoulder as the neck grows. The wavelength and amplitude of these surface oscillations are at least two order of magnitudes apart, but they are both proportional to the film thickness. Finally, we have studied experimentally the slow growth of a single crack in such polycarbonate films submitted to uniaxial and constant imposed stress. The main result of this analysis is that the instantaneous crack velocity appears to be ruled, during an experiment, by an exponential law (cf. Eq (12)) with an effective stress $\sigma_{y}^{\text {corr }}$ given by Eq. 13. Another result is the presumption that this exponential law is actually an Eyring's law. In this equation, the viscous relaxation is taken into account by the experimentally measured evolution of the ratio $\ell / \ell_{p z}$ as the crack grows. Indeed, if this ratio was constant, the stress in the plastic zone would also be constant and the velocity would increase monotonously due to the linear term in crack length. In that case, the behavior would actually be qualitatively the same as the one for crack growth in brittle facture [7. To predict fully the viscous dynamics of the crack, a second equation that will prescribe $\ell_{\mathrm{pz}}$ is missing :

$$
\frac{d \ell_{\mathrm{pz}}}{d t}=f\left(\ell_{\mathrm{pz}}, \ell, \dot{\ell}, \sigma, \ldots\right)
$$

An original theoretical approach recently developed by Bouchbinder [28] based on the Shear-Transformation-Zone Theory proposed by Falk and Langer [29] is certainly useful for deriving an equation of the plastic zone velocity (cf. Eq. (14)). Additionally, numerical simulations that can reproduce the complex visco-plastic behavior of polycarbonate may help in going further in the interpretation of our experimental results 301313233.

This work was funded with grant ANR-05-JCJC-012101. We thank K. Ravi-Chandar for insightful discussions, M. Gibert for help in PIV measurements, and C. Ybert and M. Monchanin for help in AFM imaging.

\section{Références}

1. T. C. Baker and F. W. Preston, J. Appl. Phys. 17, 170 (1946).

2. N. W. Taylor, J. Appl. Phys. 18, 943 (1947).

3. F. Bueche, J. Appl. Phys. 26, 1133 (1955) ; 28, 784 (1957).

4. S. S. Brenner, J. Appl. Phys. 33, 33 (1962).

5. S. N. Zhurkov, Int. J. Fracture Mech. 1, 311 (1965).

6. L. Pauchard and J. Meunier, Phys. Rev. Lett. 70, 3565 (1993).

7. S. Santucci, L. Vanel and S. Ciliberto, Phys. Rev. Lett. 93, 095505 (2004) ; S. Santucci, P.-P. Cortet, S. Deschanel, L. Vanel and S. Ciliberto, Europhys. Lett. 74(4), 595 (2006); S. Santucci, L. Vanel and S. Ciliberto, Eur. Phys. J. Special Topics 146, 341 (2007).

8. C. Hsieh and R. Thomson, J. Appl. Phys. 44, 2051 (1973).

9. M. Marder, Phys. Rev. E 54, 3442 (1996).

10. S. Santucci et al., Europhys. Lett. 62, 320 (2003).

11. N. Haddaoui, A. Chudnovsky and A. Moet, Polymer 27, 1377 (1986).

12. M. T. Takemory and R. P. Kambour, J. Mater. Sci. 16, 1108 (1981).

13. S. Hashemi and J. G. Williams, Plast. Rubber Compos. 29, 294 (2000).

14. R. A. Schapery, Int. J. Fracture 11, 141 (1975) ; 11, 369 (1975) ; 11, 549 (1975).

15. A. A. Kaminskii, Soviet Appl. Mech. 15, 1078 (1979); Int. Appl. Mech. 40, 829 (2004).

16. A. Chudnovsky and Y. Shulkin, Int. J. Fracture 97, 83 (1999).

17. P.-P. Cortet, L. Vanel and S. Ciliberto, Phys. Rev. Lett. 99, 205502 (2007).

18. J. Lu, and K. Ravi-Chandar, Int. J. Solids. Structures 36, 391 (1999).

19. H. Eyring, J. Chem. Phys. 4, 283 (1936).

20. C. Bauwens-Crowet, J. C. Bauwens and G. Homes, J. Mater. Sci 7, 176 (1972).

21. A. M. Donald and E. J. Kramer, J. Mater. Sci. 16, 2967 (1981).

22. G. P. Andrianova, S. A. Kechekyan and V. A. Kargin, J. Polym. Sci., Part B : Polym. Phys. 9, 1919 (1971).

23. H. Ebener, B. Pleuger and J. Petermann, J. Appl. Polym. Sci. 71, 813 (1999).

24. P.-P. Cortet, L. Vanel and S. Ciliberto, Europhys. Lett. 71(2), 1 (2005).

25. D. S. Dugdale, J. Mech. Phys. Solids 8, 100 (1960).

26. G. I. Barenblatt, Advances in Applied Mechanics (Academic, New York, 1962), Vol. 7, p.55.

27. A. Stojimirovic and A. Chudnovsky, Int. J. Frac. 57, 281 (1992).

28. E. Bouchbinder, J. S. Langer and I. Procaccia, Phys. Rev. E 75, 036107 (2007) ; 036108 (2007).

29. M. L. Falk, J. S. Langer and L. Pechenik, Phys. Rev. E 70, 011507 (2004). 
30. M. C. Boyce, E. L. Montagut and A. S. Argon, Polym. Eng. Sci. 32, 1073 (1992).

31. B. P. Gearing and L. Anand, Int. J. Solids Struct. 41, 827 (2004).

32. N. Saad-Gouider, R. Estevez, C. Olagnon and R. Séguéla, Eng. Fract. Mech. 73, 2503 (2006).

33. R. S. Hoy and M. O. Robbins, Phys. Rev. Lett. 99, 117801 (2007). 